\title{
París y el exilio de Heinrich Heine
}

\author{
Arno GIMBER \\ Departamento de Filología Alemana \\ Universidad Complutense de Madrid \\ agimber@filol.ucm.es
}

\section{RESUMEN}

La experiencia de la emigración suele ser negativa. El largo y definitivo exilio parisino de Heinrich Heine, sin embargo, no estuvo caracterizado por el deseo de volver a la patria y es, por lo tanto, atípico dentro de la historia de los exiliados alemanes. La fascinación por la metrópoli de París y una extraordinara voluntad de adaptarse a las nuevas circunstancias impidieron el surgimiento de la nostalgia, y el resultado de ello fue una fructífera mediación entre las culturas francesa y alemana por parte del poeta.

Palabras clave: Heinrich Heine, mediador, exilio, exilio alemán, París.

\section{Paris and Heinrich Heine’s exile}

\begin{abstract}
The experience of exile is often the hard experience of life abroad. But Heinrich Heine's long and final exile in Paris was not characterized by the desire to return home and is, therefore, unusual in the history of German exile. The fascination for the metropolis of Paris and an extraordinary ability to adapt to new circumstances prevented the emergence of nostalgia and the result was a successful mediation between the French and German culture.
\end{abstract}

Key words: Heinrich Heine, mediator, exile, german exile, Paris.

Je pense à mon grand cygne, avec ses gestes fous, Comme les exilés, ridicule et sublime.

(Charles Baudelaire)

\section{INTRODUCCIÓN}

Los exiliados de la Alemania nazi que llegaron hacia 1940 a México D. F., último refugio ante las redes de persecución fascista cada vez más estrechas, fundaron 
el conocido "Club Heinrich Heine" y quisieron honrar con este nombre a un exiliado que sufría como ellos la expatriación. La mayoría de ellos, Anna Seghers, Alexander Abusch, Walter Janka, Günther Ruschin o Steffi Spira, no se quedaron en México, anhelaban volver a su patria y no fueron capaces ni mostraron voluntad de integrarse en la nueva sociedad que tan generosamente les había ofrecido amparo. Ni siquiera aprendieron el idioma español para poder comunicarse correctamente con su entorno, y vivían en una especie de enclave alemán en la lejanía esperando el cambio de rumbo político en Europa para poder instalarse de nuevo en su lugar de origen.

Mal eligieron ante esta actitud estos exiliados el nombre de Heinrich Heine. Él sí se adaptó a su nueva patria. Salió en 1831 a la edad de 34 años de Hamburgo camino de París, abandonó Alemania no del todo por su propia voluntad ${ }^{1}$ pero voluntariamente se quedó en la capital francesa para el resto de su vida, es decir hasta 1856.

\section{SALIDA, CAMINO Y LLEGADA}

En primer lugar hay que insistir en que Heine se fue de Alemania sin mayores lamentos, lo que queda comprobado fijándose uno en el camino que emprendió hacia París: A mediados de abril de 1831 inició el viaje para el que su editor Campe le había regalado un diccionario alemán-francés. Salió de Hamburgo sin prisas y con desvíos llegó a París a finales de mayo. En Frankfurt, por ejemplo, posó aún para un retrato en el taller del pintor Moritz Oppenheim. Cruzó el Rin cerca de Karlsruhe y escribió al respecto en sus confesiones de 1854:

El primero de mayo de 1831 crucé el Rin. No vi al viejo dios del río, al padre Rin [...]. Estaba sentado [...] en las profundidades de sus aguas estudiando la gramática de Meidinger puesto que durante el dominio prusiano había olvidado su francés [...]. Me parecía como si conjugase: "J'aime, tu aimes, il aime, nous aimons". - ¿Pero a quién amaba? De ninguna forma a los prusianos. Vi la catedral de Estrasburgo en la lejanía, movió la cabeza como el viejo y fiel Eckart cuando percibe a un joven que va hacia la montaña de Venus. (Heine 1982: 22) ${ }^{2}$

\footnotetext{
1 Heinrich Heine era judío (aunque se convirtió en 1824 al protestantismo), y ya en el siglo XIX la situación en Alemania (para ser exacto habría que hablar de diferentes Estados dentro de la Confederación Germánica) era insoportable para esta comunidad: el edicto de los judíos de 1812, que basado en el Código Napoleónico les concedió la plena ciudadanía, fue abolido diez años más tarde, y ya en 1823 se cerraron sinagogas en varias ciudades prusianas. En este ambiente hostil no le resultó fácil a Heine ejercer su profesión de abogado y por si no fuese suficiente, su visión política liberal chocó en múltiples ocasiones con la Restauración en la Confederción Germánica.

2 "Den ersten Mai 1831 fuhr ich über den Rhein. Den alte Flußgott, den Vater Rhein, sah ich nicht [...]. Er saß [...] in der Tiefe und studierte wieder die französische Grammatik von Meidinger, weil er nämlich während der preußischen Herrschaft große Rückschritte im Französischen gemacht hatte [...]. Ich glaubte, ihn unten konjugieren zu hören: 'J'aime, tu aimes, il aime, nous aimons'. - Was aber liebte er? In keinem Fall die Preußen. Den Straßburger Münster sah ich nur von fern; er wackelte mit dem Kopfe, wie der alte getreue Eckart, wenn er einen jungen Fant erblickt, der nach dem Venusberge zieht". Todas las traducciones, si no hay otra indicación, son mías.
} 
Esta cita está llena de indirectas irónicas que tanto caracterizan a Heine: el Vater Rhein es el viejo dios fronterizo que en estos momentos estaba bajo dominio alemán, esta Alemania rancia, demasiado estrecha de la que huyó el poeta. Por ello el dios se esconde y quiere renovar sus ideas. Se prepara, estudiando lengua francesa, para una nueva vida, ahora libre y llena de amor que coincide con la visión de la montaña de Venus, un motivo romántico por excelencia y una banalización de las esperanzas más bien políticas que Heine proyectó en Francia.

Entró en París por el arco de triunfo del Boulevard Saint-Denis, en su momento erigido para honrar a Luis XIV. Ahora servía, como escribe el propio Heine, para glorificar su entrada en la capital francesa. En sus memorias (escritas veintitrés años más tarde) se acordó de la muchedumbre, vestida con elegancia como sacada de revistas de moda, con la que se encontró. Además, le impresionó que todos hablaran francés, la lengua que en Alemania era señal de un mundo distinguido, utilizada exclusivamente en los círculos cerrados de la nobleza. En Francia, sin embargo, era el idioma del pueblo entero, lo que marca aún más la frontera entre franceses y alemanes:

Los hombres estaban bien educados, las mujeres sonreían. Y si alguien me empujó sin pedirme perdón entonces estuve seguro de que de un compatriota se trataba; y si una mujer guapa resultó demasiado ácida era sin duda porque había comido demasiada col fermentada o porque era capaz de leer a Klopstock en el original. (Heine 1982: 24)

Encontrarse con compatriotas alemanes en París no era raro en la época de las revoluciones y expulsiones de la primera mitad del siglo XIX. Hay que decir que durante los duros años de la Restauración alemana (hablo de la época entre 1817 y 1848) muchos alemanes emigraron a Francia. En París, hacia 1820 con unos 800000 habitantes en total, vivían unos 7000 alemanes, en 1839 ya llegaron a 23000 y en 1846 a $59000^{4}$. Fueron los perseguidos políticos de todas las capas sociales: obreros, artesanos, artistas, editores. Entre ellos se encontraron también enseñantes y he aquí el origen de los maître de langue allemande en Francia, de los primeros profesores de lengua alemana en las escuelas públicas francesas. $Y$ es interesante, dicho sea de paso, el caso de un tal Julius Kühn que aparece en algunos listados de exiliados alemanes en París en los años treinta, y que más tarde es autor de una gramática alemana en español y el primer profesor de alemán en el Instituto de Bachillerato San Isidro en Madrid (vid. Cáceres Würsig y Marizzi 2010).

Heine evitó a los alemanes, como se infiere de la última cita, pero también porque temía (con razón) ser objetivo del espionaje de Metternich. Sin embargo, poco después de haber llegado a la "schöne Zauberstadt" (bella ciudad mágica) conoció

\footnotetext{
3 "Die Männer waren alle so höflich, und die schönen Frauen so lächelnd. Gab mir jemand unversehens einen Stoß, ohne gleich um Verzeihung zu bitten, so konnte ich darauf wetten, daß es ein Landsmann war; und wenn irgend eine Schöne etwas allzu säuerlich aussah, so hatte sie entweder Sauerkraut gegessen, oder sie konnte Klopstock im Original lesen”.

${ }^{4}$ Stierle (1998: 289) habla de hasta 100000 en 1848.
} 
en uno de los pasajes cerca del Palacio de Justicia a una vendedora de zapatos, su Mathilde, la chica que en 1841 se iba a convertir en su mujer. Dio paseos por las calles de la ciudad, frecuentó las bibliotecas y visitó los museos.

\section{EN PARÍS}

Gracias al Salón de pintura de 1831 ganó de forma inmediata su primer dinero desde el exilio. Como corresponsal mandó artículos sobre los casi mil cuadros expuestos al editor Johann Friedrich von Cotta para su publicación en el Morgenblatt für gebildete Stände de Hamburgo. Las pinturas del Salón simbolizaron para Heine la nueva libertad artística (estuvo expuesto por ejemplo La Libertad guiando al Pueblo de Delacroix), también la que había conseguido él en el sentido de poder hacer un arte autónomo ya no al servicio de los antiguos mecenas, nobleza o Iglesia. Escribió en esta dirección sobre la gran revolución que había tenido lugar en el campo de las artes. Así comenzó su trabajo como periodista cultural para revistas alemanas. Intensificaba cada vez más su actividad de autor con la editorial Cotta y con la "Augsburger Allgemeinen Zeitung" (que, dicho sea de paso, pertenecía al imperio de prensa de Cotta). En 1832 comenzó con la entrega a este periodico de los Französische Zustände, que más adelante aparecerían como libro en Hamburgo y que fueron traducidos enseguida al francés.

En ningún otro lugar de aquel entonces la independencia de los artistas había llegado tan lejos como en París. Hay que tener en cuenta que la capital francesa hacia 1830 contaba con unos 60000 abonados a periódicos (Aufenanger 2005: 24 y ss.), lo que significa en comparación con Alemania un auténico avance. A partir de 1835 Heine consiguió ganar dinero también publicando artículos en revistas francesas. El ideal romántico del artista más que en la propia Alemania, de donde surgió, se había realizado, pues, en París, la ciudad de los espíritus libres por excelencia. Sin embargo, no logró convencer con sus escritos al público francés. Por ejemplo con su artículo "État actuel de la littérature en Allemagne. De l'Allemagne depuis Madame de Staël” que apareció en la revista parisina L'Europe littéraire, intentó revisar la imagen de Alemania que en el país vecino circulaba debido al famoso libro de Madame de Staël, De l'Allemagne (1813), es decir la de una Alemania romántica de bosques misteriosos y filósofos idealistas que incluso hoy en día permanece incrustada en el imaginario nacional francés y ni los primeros escritos de Heine ni más adelante su Escuela romántica o Los dioses en el exilio consiguieron cambiar.

La situación económica de Heine en París no siempre fue muy cómoda. Recibió ingresos regulares por una herencia en Hamburgo pero la vida parisina era cara. El espía de Metternich, Eduard Beurmann, informó sobre Heine en 1837 que le gustaría volver a Alemania bajo mejores condiciones: "Despuis presque quatre ans je n'ai pas entendu un rossignol allemand. C'est assez. Je deviens triste" (Heine 1970: 96), confiesa en 1835 el poeta que en España y debido a un bon mot de Emilia Pardo Bazán es conocido como el ruiseñor de Düsseldorf.

Para no entrar en un estado melancólico o de anhelo Heine buscó contactos en la vida política, social, intelectual y artística de París y se convirtió pronto en parte 
de ella. En los salones (y no los clubes de los alemanes ${ }^{5}$ ) primero de los Saint-Simonistas y después de la Comtesse Marie d'Agoult (que residía en el Quai Voltaire número 29) contactó con los poetas, pintores y músicos más conocidos del momento: Balzac, Gauthier, Nerval, George Sand, Delacroix, Berlioz, Rossini, Chopin y Meyerbeer. Victor Hugo y Alfred de Musset no fueron de su devoción, pero Alexandre Dumas y Théophile Gautier hasta le acompañaron en su entierro el 20 de febrero de 1856 en el cementerio de Montmartre. Aparte sólo Mathilde le fue fiel hasta el final, muchos testimonios de su entorno coinciden en que el sarcasmo de Heine no resultó agradable a la mayoría de los artistas que le frecuentaban en un momento determinado de su vida parisina.

Mantuvo ciertos contactos con otros alemanes exilados: con Börne, Lassalle, Marx, Engels, Richard Wagner, pero las relaciones con ellos, siendo no del todo claras, se iban enfriando. De Karl Marx se separó y sobre Börne publicó un panfleto que demuestra que tras una primera amistad se habían convertido por discrepacias ideológicas en exiliados rivales en París.

Heine, como escritor alemán, se expresaba sobre todo en lengua alemana. Al principio hablaba un francés regular, pero poco a poco lo fue mejorando. Él mismo confesó que en los primeros meses su francés fue rudimentario, pero "tras una conversación de media hora con una joven vendedora de flores en el pasaje de l'Opéra mi francés, que desde la batalla de Waterloo se había oxidado, volvió a ser fluido, me metió tartamudeando en las conjugaciones más galantes y expliqué a la chica el sistema de Linné en el que las flores se ordenan según sus filamentos" (Heine 1982: 110) ${ }^{6}$. Muy a pesar de esta descripción jocosa, y hasta a finales de su vida, Heinrich Heine sufrió por el hecho de que el francés, tan querido por él, seguía siendo una lengua extranjera. Incluso un año antes de su muerte se sentía como un pobre ruiseñor alemán que había puesto su nido en la peluca de Voltaire (Aufenanger 2005: 145-146).

Es cierto que a veces se produjo una nostalgia hacia Alemania, sobre todo cuando se encontró, como ya hemos dicho, en precariedad económica. Pero Heine consiguió en 1840 una pensión anual de 4800 Francos del Estado francés (gracias a sus contactos en el gobierno de Thiers, que le tenía en muy alta estima y que decía que este alemán era, después de Voltaire, el francés más ingenioso) y nunca más habló del deseo de volver a su país natal.

Su relación con él fue ambigua, muchos poemas podrían demostrar su aversión política contra él, sin embargo, con la lengua fue otra cosa:

Tuve antes una patria hermosa.

El roble

\footnotetext{
${ }^{5}$ Por ejemplo existió el "Bund der Geächteten" (unión de los desterrados) que más adelante se convirtió bajo la influencia de Marx y Engels en el "Bund der Gerechten" (unión de los justos) y, a partir de 1847, en el "Bund der Kommunisten" (unión de los comunistas).

6 "Mit dem Französischen haperte es etwas bei meiner Ankunft; aber nach einer halbstündigen Unterredung mit einer kleinen Blumenhändlerin im Passage de l'Opéra ward mein Französisch, das seit der Schlacht bei Waterloo eingerostet war, wieder flüssig, ich stotterte mich wieder hinein in die galantesten Konjugationen und erklärte der Kleinen sehr verständlich das Linnéische System, wo man die Blumen nach ihren Staubfäden einteilt”.
} 
Allí crecía alto, las violetas asentían suaves -

Fue un sueño.

Me besó también en alemán y me habló en alemán

(era increíble

lo bonito que sonó) la palabra: "Te quiero" -

fue un sueño. (Heine 1979: 66)

Mandó a la prensa y a las editoriales alemanas textos sobre el progreso, la revolución y la modernidad de Francia, país en el que a veces soñó con Alemania en estos tonos altamente líricos.

\section{EL FINAL}

¿Fue París la nueva patria de Heinrich Heine o simplemente un refugio para poder vivir sin estar sometido a persecuciones? Para el poeta alemán su exilio en París fue sin duda lo primero, sobre todo porque se convirtió él mismo en parte de la vida cultural de la metrópoli francesa. Henri Heine, como le llamaron sus amigos parisinos, es el mejor ejemplo de una simbiosis franco-alemana. Como mediador que fue entre Alemania y Francia creó literatura de importancia universal.

Los últimos años de Heinrich Heine, a partir de 1848, estuvieron caracterizados por una larga y dolorosa enfermedad que le obligó a permanecer en la cama. En esta situación hubo otras muestras de nostalgia, quizá no hacia Alemania pero sin duda sí hacia sus raíces a través de cierta concienciación sobre el judaísmo. Patria, en este sentido, se podría entender no como un concepto geográfico sino religioso: la identidad judía, ignorada por Heine durante largas épocas, volvió a emerger al final de su vida en las melodías hebraicas del Romanzero y en otros poemas como por ejemplo aquél sobre la isla maravillosa llamada Bimini. Heine se apartó en sus últimos años de los reportajes y de la postura del ilustrado irónico y se acercó a lo que se podría denominar contenido y técnica musicales en la poesía. Ya en Lutezia (1840) había escrito: "La música es quizá la última palabra del arte tal y como la muerte es la última palabra de la vida"s.

En su testamento prohibió que sus restos mortales fuesen llevados a Alemania. Quiso encontrar la última morada en París, en el cementerio de Montmartre. El poema grabado en la lápida de su tumba es muy significativo: refleja la pertenencia de Heine a un mundo sin fronteras; como judío y desde muy joven había aprendido que los nacionalismos, la identificación irracional con una patria, han sido y son la peor enfermedad, la rabia nationalis, como diría Nietzsche, de nuestro mundo contemporáneo. Por ello decidió, tras 1831, no vivir nunca más en Alemania:

\footnotetext{
7 "Ich hatte einst ein schönes Vaterland. / Der Eichenbaum / wuchs dort so hoch, die Veilchen nickten sanft - / es war ein Traum. // Das küßte mich auch deutsch und sprach auf deutsch / (man glaubt es kaum, / wie gut es klang) das Wort: ,Ich liebe dich“ - / es war ein Traum”.

8 "Die Musik ist vielleicht das letzte Wort der Kunst, wie der Tod das letzte Wort des Lebens".
} 
Dónde podrá decir el trotamundos que halló por fin su último descanso? ¿En el sur, frente al mar, bajo palmeras? ¿O bajo tilos junto al Rin, tan manso?

¿Qué extranjero me hará la caridad de una tumba, y en qué desierto extraño? ¿O quedaré tirado en una playa de aún no sé qué mar del desengaño?

¡Qué más da! Caiga donde caiga ha de haber cielo, y estará estrellado. Además, como yo ya no seré mi cuerpo, el dónde me trae sin cuidado. (Balzer 1995: 215) ${ }^{9}$

\section{BIBLIOGRAFÍA}

AUFENANGER, Jörg (2005): Heinrich Heine in Paris. Múnich: Deutscher Taschenbuchverlag.

BALZER, Berit (1995): Heinrich Heine. Gedichte - Auswahl. Antología poética. Madrid: Ediciones de la Torre.

BETZ, Louis P. (1894): Heine in Frankreich. Eine literaturhistorische Untersuchung. Zürich: A. Müller.

CÁCERES WÜRSIG, Ingrid; y MARIZZI, Bernd (2010): "La Academia Alemana-Española de 1840 de Julio Kühn: relato de un proyecto de colaboración científica y cultural". Estudios Filológicos Alemanes, núm. 20, pp. 415-436.

GÜNTHER, Herbert (1979): Deutsche Dichter erleben Paris. Uhland. Heine. Hebbel. Wedekind. Dauthendey. Holz. Rilke. Zweig. Pfulingen: Günther Neske.

HEINE, Heinrich (1970): Briefe 1831-1841. Berlín: Akademie-Verlag (=Säkularausgabe).

- (1979): Gedichte 1827-1844 und Versepen. Berlín: Akademie-Verlag (=Säkularausgabe).

- (1982): "Geständnisse”, in Historisch-kritische Gesamtausgabe der Werke. Düsseldorfer Ausgabe, Manfred Windfuhr (ed.), tomo 15 (Geständnisse, Memoiren, Kleinere autobiographische Schriften). Hamburg: Hoffman und Campe.

HÖHN, Gerhard; \& LIEDTKE, Christian (eds.) (2010): Auf der Spitze der Welt. Mit Heine durch Paris. Hamburg: Hoffmann und Campe.

KRUSE, Joseph A.; \& WERNER, Michael (1981): Heine in Paris 1831-1856. Düsseldorf: Droste.

PAWEL, Ernst (1997): Der Dichter stirbt, Heinrich Heines letzte Jahre in Paris. Berlín: Berlin Verlag.

STIERLE, Karlheinz (1998): Der Mythos von Paris. Zeichen und Bewußtsein der Stadt. Múnich: Deutscher Taschenbuchverlag.

\footnotetext{
9 "Wo wird einst des Wandermüden / letzte Ruhstätte sein? / Unter Palmen in dem Süden? / Unter Linden an dem Rhein? // Werd' ich wo in einer Wüste / Eingescharrt von fremder Hand? / Oder ruh' ich an der Küste / Eines Meeres in dem Sand? // Immerhin! Mich wird umgeben / Gotteshimmel, dort wie hier, / Und als Totenlampen schweben / Nachts die Sterne über mir”. (Balzer 1995: 215).
} 